\title{
An overview on Pharmacokinetics of Polymeric Nanoparticles Intended for Oral Delivery
}

\author{
Srinu Naik Sapavatu', Ravikanth Chinthala², Rajendra Kumar Jadi²,* \\ ${ }^{1}$ Department of Chemical Engineering, University College of Technology, Osmania University, Hyderabad, Telangana, INDIA. \\ ${ }^{2}$ Department of Pharmacy, University College of Technology, Osmania University, Hyderabad, Telangana, INDIA.
}

\begin{abstract}
Polymeric nanoparticles are investigated as drug delivery devices to overcome physicochemical and pharmacokinetic (PK) problems. Many drugs were found to be drug-like during the pharmacological screening. However, all of them cannot make it to the market due to toxicity and poor bio-availability. The advent of nanoparticles has opened a new window of research where poorly soluble drugs are made useful. Nanoparticles have also been developed for favourable pharmacokinetics to avoid toxicity and side effects, to target the desired site of action and to provide triggered drug release. Poorly soluble drugs can be entrapped, encapsulated, coated or chemically bound in nanoparticles to deliver the drugs. Generally, in standard drug delivery, devices carry the drug until the gastrointestinal tract (GIT) and the drug is released, but the tool never expected to be entering the blood. However, in polymeric nanoparticles, sometimes the drug is being carried to the blood and the site of action. Polymeric nanoparticles release the drug in the gastrointestinal tract (GIT) or enter the GIT through
\end{abstract}

various pathways to appear in the bloodstream. In this review, we discuss the fate of polymeric nanoparticles made up of biodegradable polymers after they are administered through the oral route.

Key words: Nanoparticle, Pharmacokinetics, Drug delivery, Polymer, Oral route.

\section{Correspondence}

Mr. Jadi Rajendra Kumar,

Department of Pharmacy, University College of Technology, Osmania University, Hyderabad-500 007, Telangana, INDIA.

Phone: +91 040-2768 2444

Email: rajendra.rajaji@gmail.com

DOI: 10.5530/jyp.2020.12.57

\section{INTRODUCTION}

The classical oral drug delivery system breakdown in the gastrointestinal tract (GIT) and release the drug. After the drug release in the GIT, the drugs penetrate through biological barriers and reach the site of action. But with nanoparticles, the drug goes into the bloodstream, acting as drug delivery systems that can improve the bioavailability of the drugs. ${ }^{1}$ In drug targeting, nanoparticles are expected to be accumulated in high concentrations at the site of action when compared to other tissues. ${ }^{2}$ After accumulation, the drug will be released at the target organ broadening the therapeutic window. Due to the broad therapeutic window, the drug's inherent toxicity will be reduced. It helps in the improvement of the bioavailability of the drugs that have poor bioavailability (BA). There may be various reasons for poor BA, like poor solubility, reduced permeability, or poor stability. All these issues can be avoided with the nanoparticle approach.

Nanoparticles have been developed for all types of drug molecules, like those having excellent physicochemical properties or good BA. This is because the nanoparticles can alter the pharmacokinetics of the drugs and can result in favourable kinetics of the drugs to avoid toxicity. One such example is doxorubicin nanoparticles. Doxorubicin has excellent physicochemical properties, but the distribution of doxorubicin to the heart had produced cardiac ailments. This is avoided by entrapping or encapsulating the doxorubicin in nanoparticles. ${ }^{3}$ The classical pharmacokinetic studies which measure the drug levels in the blood might not have taken into consideration of unreleased drug from nanoparticles. Many pharmacokinetic studies presume that the drug in nanoparticles is released in the bloodstream, which is not the case in reality. ${ }^{4}$
In this review, we collected the information from various studies related to the pharmacokinetic concepts like absorption, distribution, metabolism and excretion of polymeric nanoparticles.

\section{ABSORPTION OF NANOPARTICLES}

Gastrointestinal Tract (GIT) presents exciting anatomy and physiology for absorption, as it is made up of the absorption of nutrients. The large surface area of the intestine, varying $\mathrm{pH}$ through the GIT and the presence of Peyer's patches made it more attractive for the absorption of nutrients and solubilized drugs. While presenting nanoparticles for oral intake, all these variations of the GIT might have to be considered. The external layer is the mucus layer, which acts as the first barrier for nanoparticles that need to be crossed. Nanoparticles also developed for targeting the mucus layer for better absorption. ${ }^{5}$ After the mucus layer, appears the real membrane of epithelial cells. Nanoparticles need to be absorbed intact and the barrier might not allow this to be happening. ${ }^{6,7}$ The understanding so far is not clear and some researchers suggest that the nanoparticles are absorbed through $\mathrm{M}$ cells of the Peyer's patches and some assume that nanoparticles are taken up from the healthy gastric epithelial cells. ${ }^{8-11}$ The data on the pharmacokinetics of the orally delivered nanoparticles is not extensive enough to conclude. Generally, in vitro, studies are carried out to see if the nanoparticles can penetrate the epithelium of the GIT. Most of the time, in vitro studies, are not able to be correlated with the in vivo studies.

Mucus on the lining of gastric epithelium plays an essential role in the absorption of nanoparticles or drugs released from the nanoparticles. ${ }^{12}$ Nanoparticles that adhere to the mucus for sustained release of the drug are known as mucoadhesive nanoparticles. ${ }^{13}$ Mucoadhesive

This is an open access article distributed under the terms of the Creative Commons Attribution-NonCommercial-ShareAlike 4.0 License, which allows others to remix, tweak, and build upon the work non-commercially, as long as the author is credited and the new creations are licensed under the identical terms. 
nanoparticles were meant to be releasing the drug in the GIT, whereas mucus penetrating nanoparticles can get through the GIT intact. Mucoadhesive nanoparticles were explored extensively for the prolonged release of the drug-using various types of polymers. ${ }^{14}$ Among them and thiolated polymers were adhering to the mucus firmly. ${ }^{15}$ However, later, mucoadhesive nanoparticles were understood to be affecting the mucus barrier properties. ${ }^{16}$ Currently, mucus penetrating nanoparticles were thought to be suitable carriers of drugs through GIT and other barriers where mucus plays an important role. ${ }^{12}$ PEGylation is the modification of polymers or nanoparticles with chemically attaching or coating the surface with PEG. PEGylation makes them suitable for various drug delivery applications like high circulation, mucus penetration and protection from the mononuclear phagocyte system (MPS) of the body. In oral delivery, PEGylation helps in the penetration of nanoparticles through the mucus barrier. ${ }^{17}$

Nanoparticles are absorbed intact or can be adsorbed onto mucus to release the drug. They can get absorbed by various pathways. In a recent study, authors have found out that the nanoparticles absorbed into both lymph and blood circulation from the GIT. It can increase the BA of the drug-loaded onto them. ${ }^{18}$ The size of those nanoparticles was around $170 \mathrm{~nm}$ and is positively charged. There were reports of nanoparticles absorbed with $250 \mathrm{~nm}$ size and negatively charged. ${ }^{19}$ Particle size more than $500 \mathrm{~nm}$, considered to be not absorbable from the GIT. ${ }^{20}$ The Surface charge plays a vital role in the absorption of nanoparticles and non-ionic nanoparticles showed significant improvements in the uptake of nanoparticles. ${ }^{8}$ Some researchers have developed inert peptide sequences using phage display to target $M$ cells and epithelial cells of the intestine to increase the absorption. ${ }^{21}$ The particle geometry also influences the absorption and particles with high aspect ratio and rodshaped nanoparticles better absorbed than spherical nanoparticles. ${ }^{6}$ The parameters were affecting absorption shown in Table 1.

\section{DISTRIBUTION OF NANOPARTICLES}

Distribution patterns of drug-loaded nanoparticles delivered through various routes vary for each way. ${ }^{22}$ Orally given nanoparticles distribution is different from intravenously delivered nanoparticles. ${ }^{23}$ Most of the distribution or pharmacokinetic studies have been carried out after intravenous administration-very few studies conducted for orally delivered nanoparticles. And the effect of the size, surface charge, percentage drug loading, detection methods have been very different for different studies. Thus, making out a conclusion is not that easy. Majority of the reviews were done based on the analysis of the drug or a label rather than the nanoparticle as a whole or material which is used for the preparation of nanoparticles. ${ }^{24}$

Nanoparticles are known to be captured by the MPS. ${ }^{25}$ To avoid accumulation in organs of MPS, generally, particles are coated with or chemically bound with PEG (Polyethylene glycol). This PEGylation showed to possess many pros in the case of the nanoparticles. The nanoparticles alter the drug's distribution pattern in many cases. The release rate from the nanoparticles and the circulation times of the nanoparticles increase the BA. The nanoparticles, if they targeted to be released only at the desired site, the therapeutic window rises, making the drugs safer.

The outcome of nanoparticles based on the distribution during the targeting, the targeting is known to be two types, i.e., passive targeting and active targeting. Passive targeting utilizes the physiology of the diseased parts and active targeting utilizes surface coatings or chemically attached ligands on nanoparticles. These nanoparticles are tailor-made to suit the requirements. The blood circulation and lymphatic circulation both play a crucial role in the distribution of nanoparticles. Most of the uncoated nanoparticles reach the MPS organs cleared from the circulation.
Surface coating or chemical attachment with PEG, which is known as PEGylation, improves the blood circulation times of nanoparticles, this is helpful in the extended-release (ER) of the drugs. ${ }^{24}$

In a study, researchers have used a physiologically based pharmacokinetic (PBPK) model with prepared with PLGA (poly-lactic-co-glycolic acid) nanoparticles and modified with PEG. They conclude that PBPK model can be utilized to understand the polymeric nanoparticle distribution and their relationship with the physicochemical properties. ${ }^{9}$

The BA is defined as the rate and extent at which the drug reaches systemic circulation. In a real sense, the free drug that is available at the site of action is BA. However, most of the pharmacokinetics has analyzed the drug concentrations in the blood without differentiating the free and nanoparticle bound drug. The BA of the drugs carried by the nanoparticles shown to be increasing many folds. ${ }^{26-30}$ The BA concept, blood levels will not be corroborated to the effect when the nanoparticles are developed for targeting specific organs or tissues in the body. In targeted nanoparticles, the drug might not appear in reasonable quantities in the blood, but the action would be mightier than the free drug. Probably new approaches in pharmacokinetics are needed for understanding the kinetics of drug release and reaction from nanoparticles.

\section{METABOLISM OF NANOPARTICLES}

Drugs that are entrapped in the nanoparticles are protected from metabolism for longer durations if they are long-circulating. Nanoparticles that are not coated with PEG or any other material that can protect nanoparticles from opsonisation are accumulated in the organs of MPS. These are found mostly in the spleen, liver and lungs. The lymphatic absorption of the nanoparticles avoids the first-pass metabolism that is experienced by the naked drugs absorbed through GIT epithelium. Nanoparticles, by avoiding the first-pass metabolism, supply the drug to the target tissues in more enormous amounts. This is more relevant to lipid-based Nano systems like Nano emulsions and solid lipid nanoparticles. ${ }^{26}$

The clearance from the body is delayed in overall with the nanoparticles after oral administration. ${ }^{31}$ Due to this, the drug-exposed for longer durations resulting in potency and or lesser dosage frequencies. This could be because of the drug in nanoparticles is not free and not available for metabolism.

\section{EXCRETION OF NANOPARTICLES}

Nanoparticles that are less than the size of $5 \mathrm{~nm}$ are known to be excreted from the kidneys. ${ }^{31,32}$ But most of the time, nanoparticles made up of polymers are generally larger and are not discharged directly. This is the reason for the increased circulation times and reduced clearance of polymeric nanoparticles. Most of the polymeric nanoparticles are excreted through faces after metabolism in the liver and other tissues. ${ }^{10,33,34}$ However, the excretion of polymeric nanoparticles still needs to be studied and understood. The use of biodegradable materials

Table 1: Absorption of nanoparticles from the gastrointestinal tract.

\begin{tabular}{ccc}
\hline Parameter & Quality & Outcome \\
\hline Shape & High aspect ratio & Better lymphatic absorption \\
Surface charge & Non-ionic & Better absorption $^{8}$ \\
Surface modifications & PEGylation & Increased absorption $^{17}$ \\
Size & $<500 \mathrm{~nm}$ & Absorbed through GIT \\
Surface ligands & Inert peptides & Increased absorption \\
Surface modifications & Thiolation & Mucoadhesion $^{22}$ \\
\hline
\end{tabular}


helps in the clearance of the body. Non-biodegradable materials need to be used with caution as they can reside for years in the body. ${ }^{31}$

\section{CONCLUSION}

When analyzing the pharmacokinetics of the drugs entrapped in the nanoparticles, generally, the drug in the nanoparticles in the blood is also estimated because of the unfortunate separation of nanoparticles from the blood. Meaning that the released drugs over estimated to be free from the nanoparticles while the drug is still in the nanoparticles. The techniques currently used by the researchers generally are not considering the drug release from the nanoparticles. The kinetics might be well understood when the concentrations of free drug and drug entrapped, encapsulated, or coated are analyzed separately. The pharmacokinetics of nanoparticles is not well defined and need more studies in this area.

\section{ACKNOWLEDGEMENT}

The authors acknowledge University College of Technology, Osmania University, Hyderabad for their continuous support and encouragement.

\section{CONFLICT OF INTEREST}

The authors declare no conflict of interest.

\section{ABBREVIATIONS}

BA: Bioavailability; GIT: Gastrointestinal tract; MPS: Mononuclear phagocytic system; PBPK: Physiologically based pharmacokinetic; PLGA: Poly (lactic-co-glycolic acid); PEG: Polyethylene glycol.

\section{REFERENCES}

1. Devadasu VR, Bhardwaj V, Kumar MR. Can controversial nanotechnology promise drug delivery?. Chem Rev. 2013;113(3):1686-735.

2. Yun Y, ChoYW, Park K. Nanoparticles for oral delivery: targeted nanoparticles with peptidic ligands for oral protein delivery. Adv Drug Deliv Rev. 2013;65(6):822-32

3. Kalaria DR, Sharma G, Beniwal V, Kumar MR. Design of biodegradable nanoparticles for oral delivery of doxorubicin: In vivo pharmacokinetics and toxicity studies in rats. Pharm Res. 2009;26(3):492-501.

4. Rodallec A, Benzekry S, Lacarelle B, Ciccolini J, Fanciullino R. Pharmacokinetics variability: Why nanoparticles are not just magic-bullets in oncology. Crit Rev Oncol Hematol. 2018;129:1-2.

5. Anderski J, Mahlert L, Mulac D, Langer K. Mucus-penetrating nanoparticles: Promising drug delivery systems for the photodynamic therapy of intestinal cancer. Eur J Pharm Biopharm. 2018;129:1-9.

6. Li D, Zhuang J, He H, Jiang S, Banerjee A, Lu Y, et al. Influence of particle geometry on gastrointestinal transit and absorption following Oral administration. ACS Appl Mater Interfaces. 2017;9(49):42492-502.

7. Rao DA, Forrest ML, Alani AW, Kwon GS, Robinson JR. Biodegradable PLGA based nanoparticles for sustained regional lymphatic drug delivery. J Pharm Sci. 2010;99(4):2018-31.

8. Du XJ, Wang JL, labal S, Li HJ, Cao ZT, Wang YC, Du JZ, et al. The effect of surface charge on oral absorption of polymeric nanoparticles. Biomater Sci. 2018;6:642-50

9. Li M, Panagi Z, Avgoustakis K, Reineke J. Physiologically based pharmacokinetic modeling of PLGA nanoparticles with varied $\mathrm{m}-\mathrm{PEG}$ content. Int J Nanomedicine. 2012;7:1345-56

10. Bergin IL, Witzmann FA. Nanoparticle toxicity by the gastrointestinal route: Evidence and knowledge gaps. Int J Biomed Nanosci Nanotechnol. 2013;3(12):163.

11. Nair S, Sasidharan A, Rani VD, Menon D, Nair S, Manzoor K, et al. Role of size scale of $\mathrm{ZnO}$ nanoparticles and microparticles on toxicity toward bacteria and osteoblast cancer cells. J Mater Sci Mater Med. 2009;20:S235-41.

12. Ensign LM, Cone R, Hanes J. Oral drug delivery with polymeric nanoparticles: The gastrointestinal mucus barriers. Adv Drug Deliv Rev. 2012;64(6):557-70.

13. Fabiano A, Piras AM, Uccello-Barretta G, Balzano F, Cesari A, Testai L, et al. Impact of mucoadhesive polymeric nanoparticulate systems on oral bioavailability of a macromolecular model drug. Eur J Pharm Biopharm. 2018;130:281-9.

14. Charlie-Silva I, DeMelo NF, Gomes JM, Fraceto LF, DeMelo DC, DeOliveira SJ, et al. Nanoparticle mucoadhesive system as a new tool for fish immune system modulation. Fish Shellfish Immunol. 2018:80:651-4.

15. Arif M, Dong QJ, Raja MA, Zeenat S, Chi Z, Liu CG. Development of novel pHsensitive thiolated chitosan/PMLA nanoparticles for amoxicillin delivery to treat Helicobacter pylori. Mater Sci Eng C Mater Biol Appl. 2018;83:17-24.

16. Wang YY, Lai SK, So C, Schneider C, Cone R, Hanes J. Mucoadhesive nanoparticles may disrupt the protective human mucus barrier by altering its microstructure. PLoS One. 2011;6(6):e21547.

17. Huckaby JT, Lai SK. PE Gylation for enhancing nanoparticle diffusion in mucus. Adv Drug Deliv Rev. 2018;124:125-39.

18. Ren $T$, Wang $Q$, Xu Y, Cong L, Gou J, Tao $X$, et al. Enhanced oral absorption and anticancer efficacy of cabazitaxel by overcoming intestinal mucus and epithelium barriers using surface polyethylene oxide (PEO) decorated positively charged polymer-lipid hybrid nanoparticles. J Control Release. 2018;269:423-38.

19. Devadasu VR, Wadsworth RM, Kumar MR. Tissue localization of nanoparticles is altered due to hypoxia resulting in poor efficacy of curcumin nanoparticles in pulmonary hypertension. Eur J Pharm Biopharm. 2012;80(3):578-84.

20. BorelT, Sabliov CM. Nanodelivery of bioactive components for food applications: types of delivery systems, properties, and their effect on ADME profiles and toxicity of nanoparticles. Annu Rev Food Sci Technol. 2014;5:197-213.

21. Fievez V, Plapied L, Plaideau C, Legendre D, DesRieux A, Pourcelle V, et al. In vitro identification of targeting ligands of human $\mathrm{M}$ cells by phage display. Int $J$ Pharm. 2010;394(1-2):35-42.

22. Dünnhaupt S, Barthelmes J, Hombach J, Sakloetsakun D, Arkhipova V, BernkopSchnürch A. Distribution of thiolated mucoadhesive nanoparticles on intestinal mucosa. Int J Pharm. 2011;408(1-2):191-9.

23. Simon LC, Sabliov CM. The effect of nanoparticle properties, detection method delivery route and animal model on poly (lactic-co-glycolic) acid nanoparticles biodistribution in mice and rats. Drug Metab Rev. 2014;46(2):128-41.

24. He H, Jiang S, Xie Y, LuY, Qi J, Dong $X$, et al. Reassessment of long circulation via monitoring of integral polymeric nanoparticles justifies a more accurate understanding. Nanoscale Horiz. 2018;3(4):397-407.

25. Owens III DE, Peppas NA. Opsonization, biodistribution and pharmacokinetics of polymeric nanoparticles. Int J Pharm. 2006:307(1):93-102.

26. Yao M, McClements DJ, Xiao H. Improving oral bioavailability of nutraceuticals by engineered nanoparticle-based delivery systems. Curr Opin Food Sci. 2015;2:14-9

27. Anwar M, Warsi MH, Mallick N, Akhter S, Gahoi S, Jain GK et al. Enhanced bioavailability of nano-sized chitosan-atorvastatin conjugate after oral administration to rats. Eur J Pharm Sci. 2011;44(3):241-9.

28. Mutalik S, Anju P, Manoj K, Usha AN. Enhancement of dissolution rate and bioavailability of aceclofenac: A chitosan-based solvent change approach. Int J Pharm. 2008;350(1-2):279-90.

29. Solas M, Martínez-Ohárriz MC, Muñoz E, Santos-Magalhães NS, Ramirez MJ, Irache JM. Pegylated nanoparticles for the oral delivery of nimodipine: Pharmacokinetics and effect on the anxiety and cognition in mice. Int J Pharm. 2018;543(1-2):245-56

30. Yoshioka C, Ito $Y$, Nagai N. An oral formulation of cilostazol nanoparticles enhances intestinal drug absorption in rats. Exp Ther Med. 2018;15(1):454-60.

31. Kadam RS, Bourne DW, Kompella UB. Nano-advantage in enhanced drug delivery with biodegradable nanoparticles: Contribution of reduced clearance. Drug Metab Dispos. 2012;40(7):1380-8.

32. Choi HS, Liu W, Misra P, Tanaka E, Zimmer JP, Ipe BI, et al. Renal clearance of quantum dots. Nat Biotechnol. 2007;25(10):1165-70.

33. Wu T. Tang M. Review of the effects of manufactured nanoparticles on mammalian target organs. J Appl Toxicol. 2018;38(1):25-40.

34. Song X, Zhao X, Zhou Y, Li S, Ma Q. Pharmacokinetics and disposition of various drug loaded biodegradable poly (lactide-co-glycolide)(PLGA) nanoparticles. Curr Drug Metab. 2010;11(10):859-69.

Article History: Submission Date : 09-03-2020; Revised Date : 16-05-2020 ; Acceptance Date : 21-06-2020

Cite this article: Sapavatu SN, Chintala R, Jadi RK. An overview on Pharmacokinetics of Polymeric Nanoparticles Intended for Oral Delivery. J Young Pharm. 2020;12(3):187-9 